\title{
TOF-SIMS: Accurate Mass Scale Calibration
}

\author{
F. M. Green, I. S. Gilmore, and M. P. Seah \\ Quality of Life Division, National Physical Laboratory, Middlesex, United Kingdom
}

\begin{abstract}
A study is presented of the factors affecting the calibration of the mass scale in time-of-flight secondary ion mass spectrometry (TOF-SIMS). At the present time, TOF-SIMS analysts using local calibration procedures achieve a rather poor relative mass accuracy of only $150 \mathrm{ppm}$ for large molecules $(647 \mathrm{u})$ whereas for smaller fragments of $<200 \mathrm{u}$ this figure only improves to $60 \mathrm{ppm}$. The instrumental stability is $1 \mathrm{ppm}$ and better than $10 \mathrm{ppm}$ is necessary for unique identification of species. The above experimental uncertainty can lead to unnecessary confusion where peaks are wrongly identified or peaks are ambiguously assigned. Here we study, in detail, the instrumental parameters of a popular single stage reflection TOF-SIMS instrument with ion trajectory calculations using SIMION. The effect of the ion kinetic energy, emission angle, and other instrumental operating parameters on the measured peak position are determined. This shows clearly why molecular and atomic ions have different relative peak positions and the need for an aperture to restrict ions at large emission angles. These data provide the basis for a coherent procedure for optimizing the settings for accurate mass calibration and rules by which calibrations for inorganics and organics may be incorporated. This leads to a new generic set of ions for mass calibration that improves the mass accuracy in our interlaboratory study by a factor of 5 . A calibration protocol is developed, which gives a relative mass accuracy of better than $10 \mathrm{ppm}$ for masses up to $140 \mathrm{u}$. The effects of extrapolation beyond the calibration range are discussed and a recommended procedure is given to ensure that accurate mass is achieved within a selectable uncertainty for large molecules. Additionally, we can alternatively operate our instrument in a regime with good energy discrimination (i.e., poor energy compensation) to study the fragmented energies of molecules. This leads to data that support previous concepts developed in G-SIMS. (J Am Soc Mass Spectrom 2006, 17, 514-523) (C 2006 American Society for Mass Spectrometry
\end{abstract}

$O^{2}$ tatic SIMS (SSIMS) is a powerful technique for the analysis of organic and molecular surfaces. Over the last decade, instrumentation has improved significantly so that modern instruments now have very high reliability. Together with reference procedures and methods, the intensity repeatability has improved by over a factor of 10. In a recent VAMAS interlaboratory study [1] conducted by NPL, over $84 \%$ of instruments exhibited excellent intensity repeatabilities of better than $1.9 \%$. It was also demonstrated that the comparability of relative ion intensities between different instrument designs can be $<4 \%$ by incorporation of the concept of the relative instrument spectral response (RISR) function [1].

A significant issue for many analysts is establishing an accurate calibration of the mass scale for time-of-flight (TOF) instruments. In a recent ISO [2] survey of needs for standardization in static SIMS, analysts ranked a procedure for mass calibration as the top priority. Typically, this needs to be conducted for each spectrum since small variations in the sample height lead to significant differences in the arrival times of secondary ions. The calibration of the mass scale is essential even for simple comparison between spectra and for peak identification. An

Published online February 28, 2006

Address reprint requests to F. M. Green, Quality of Life Division, National Physical Laboratory, Hampton Road, Teddington, Middlesex TW11 0LW, UK. E-mail: Felicia.Green@npl.co.uk increasing requirement is the identification of the chemical composition from an accurate measurement of the mass of the fragment ion. Figure 1 illustrates the required relative mass accuracy to do this and to distinguish between molecules that contain different chemical constituents, such as $\mathrm{CH}_{2}$ or $\mathrm{N}$, but are of the same nominal mass rounded to the nearest integer mass (here, $14 \mathrm{u}$ ) in the mass range 0 to $1000 \mathrm{u}$. Here we use the unified atomic mass unit with symbol " $u$ ". Clearly, a relative mass accuracy of at least $10 \mathrm{ppm}$ is required to distinguish between $\mathrm{C}_{2} \mathrm{H}_{4}(28.03130 \mathrm{u})$ and $\mathrm{Si}(27.97692 \mathrm{u})$ in parent ions with total mass up to $1000 \mathrm{u}$ and between $\mathrm{CH}_{2}$ $(14.01565 \mathrm{u})$ and $\mathrm{N}(14.00307 \mathrm{u})$ in parent ions with total mass up to $300 \mathrm{u}$. To keep terms clear, we define the mass accuracy, $\Delta M$, as the difference between the measured peak mass $M_{\mathrm{P}}$ and the true mass $M_{\mathrm{T}}$

$$
\Delta M=M_{\mathrm{P}}-M_{\mathrm{T}}
$$

and the relative mass accuracy $W$ is given by

$$
W=\frac{\Delta M}{M}
$$

In the text that follows $W$ will be given in parts-permillion (ppm).

To evaluate the present errors in the calibration of the mass scale, participants in the VAMAS TOF-SIMS 


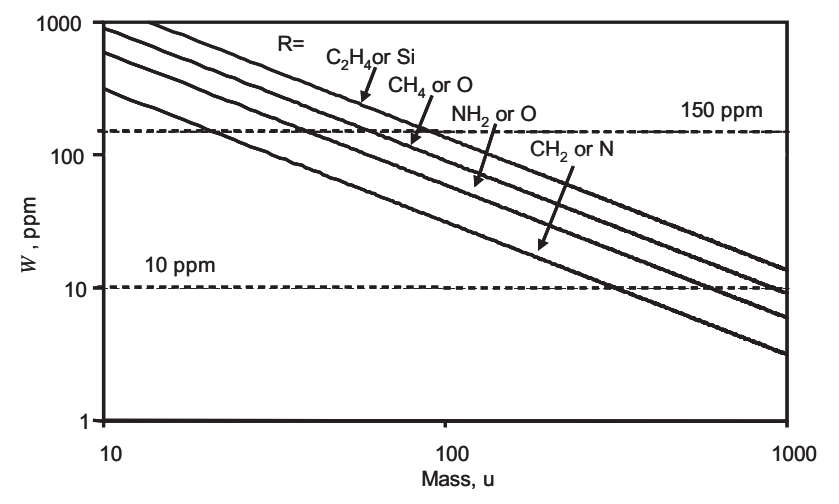

Figure 1. The required relative mass accuracy, $W$, in ppm to distinguish between ions including the functional groups, $\mathrm{R}$, where the alternatives for $\mathrm{R}$ (e.g., $\mathrm{CH}_{2}$ or $\mathrm{N}$ ) have the same nominal mass.

interlaboratory study $[1,3]$ were asked to calibrate their mass spectra using their local procedures [Gilmore, I. S.; Green, F. M.; Seah, M. P. Static TOF-SIMS - A VAMAS Interlaboratory Study. Part II, to be published]. Data from 32 TOF-SIMS instruments were submitted. Details of the instruments, reference materials, and measurement protocols are given elsewhere $[1,3]$. Figure 2 shows the mass accuracy $\Delta M$ of the molecular ion for Irgafos (an additive to the PC reference material) for the 32 instruments. Clearly, $\Delta M$ is, on average, $0.065 \mathrm{u}$ below the true mass, so that $W$ is $-150 \mathrm{ppm}$. This is poor compared with the requirement for identification. It is clear from Figure 1 that a relative mass accuracy of $150 \mathrm{ppm}$ is unacceptable even for basic differentiation between ions that vary in constituents by, say, $\mathrm{Si}$ instead of $\mathrm{C}_{2} \mathrm{H}_{4}$ above $90 \mathrm{u}$. The error bars in Figure 2 show the one sigma standard deviation of five repeat measurements in each laboratory. Ignoring the four laboratories with very poor repeatabilities, the average is $0.0124 \mathrm{u}$, and shows that instrument control would generally be adequate for precise measurements if there were no biases in the data.

In this paper, we develop a systematic approach to identifying the cause of mass inaccuracies, to optimizing instrument parameters to improve accuracy, and to provide guidance and recommendations for practical analysts.

There have been surprisingly few studies of the mass-scale calibration in TOF-SIMS [4-6]. This may be because in the past, other issues such as poor repeatability dominated accuracy. These are now under much better control [1].

Niehuis et al. [4] used a single stage reflection time-of-flight analyzer to illustrate the benefits of improved mass resolution and to study the mass scale accuracy using a trimethyl silylated silicon oxide surface. They found an average value for $W$ of $20 \mathrm{ppm}$ for 26 negative ions with masses between 60 and $149 \mathrm{u}$. It was noted that the mass calibration depends on the differences in the energy distribution of the secondary ion species. It was estimated that a deviation of $<10 \mathrm{ppm}$ was expected for a $1.5 \mathrm{eV}$ change of the energy distribution. They also noted that the accuracy of the time-to-digital converter (TDC) clock was a significant factor. For their instrument, the clock accuracy was quoted as \pm 100 ps which, they note, gives a mass deviation of $\pm 7.5 \mathrm{ppm}$ at $110 \mathrm{u}$. This degrades linearly as the mass increases. In a study [5] of polymers PDMS and Nylon 66, they showed an average deviation in the low mass range $(<100 \mathrm{u})$ of $\sim 20 \mathrm{ppm}$, and this is improved to $10 \mathrm{ppm}$ with a TDC operating with $156 \mathrm{ps} /$ channel.

The rule-of-thumb is that inorganics and organics should not be combined in calibration. Reichlmaier et al. [6] conducted a detailed assessment of the accuracy of mass scale calibration and of the repeatability over a period of 6 months. They used a PHI 7210 TOF spectrometer with a two stage reflection energy compensator to study the effect of calibration with different sets of ions on both the average mass accuracy and the repeatability over 34 spectra recorded in that period. They showed that, with a mass calibration using the 3 peaks for $\mathrm{CH}_{3}^{+}, \mathrm{C}_{2} \mathrm{H}_{3}^{+}$, and $\mathrm{C}_{3} \mathrm{H}_{5}^{+}$, an average $W$ of $10.4 \mathrm{ppm}$ was achieved for 7 further peaks in the mass range 104 to $577 \mathrm{u}$ for poly(ethylene terephthalate). By adding the 3 calibration peaks for $\mathrm{C}_{4} \mathrm{H}_{7}^{+}, \mathrm{C}_{6} \mathrm{H}_{5}^{+}$, and $\mathrm{C}_{4} \mathrm{H}_{7} \mathrm{O}^{+}$, the average value of $W$ improved to the excellent value of $5.3 \mathrm{ppm}$. To investigate the effect of different energy distributions between atomic and molecular ions, they studied the corresponding negative ion spectra. The average repeatability of 12 ions between 12 and $357 \mathrm{u}$ calibrated with atomic ions $\mathrm{C}^{-}, \mathrm{O}^{-}, \mathrm{Cl}^{-}$, and molecular ions $\mathrm{CH}^{-}, \mathrm{C}_{2} \mathrm{H}_{4}^{-}$, and $\mathrm{C}_{4} \mathrm{H}^{-}$showed no perceptible difference, being 14 and $16 \mathrm{ppm}$, respectively. This could be, as we shall see later, that the small organic ions that result from significant rearrangement have broader energy distribution and higher energy maxima than a corresponding ion with the same number of atoms but arising with fewer rearrangements. For example, clearly $\mathrm{C}_{4} \mathrm{H}^{-}$in general will have suffered significant changes compared with, say, $\mathrm{C}_{4} \mathrm{H}_{7}^{-}$. Reichl-

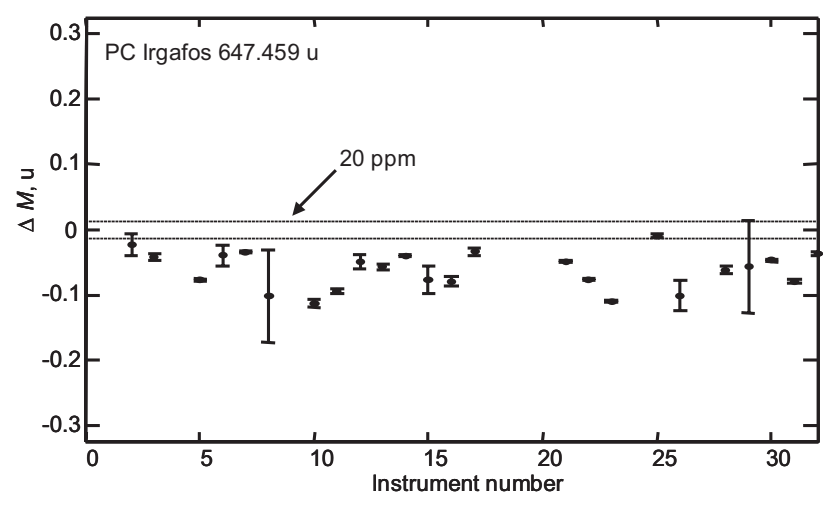

Figure 2. Mass accuracy, $\Delta M$, of the protonated Irgafos molecular ion for instruments with reasonable quality data from 32 instruments in an interlaboratory study [1]. The error bars show the standard deviations of the five repeat measurements in each laboratory. Also shown are two dashed lines indicating the requirement for $\pm 20 \mathrm{ppm}$ relative accuracy. 
maier et al. [6] clearly demonstrate the need for sufficient counts in a peak for accurate mass assignment. They conclude that $>1000$ counts are required for $W<10 \mathrm{ppm}$ and that, over the range $0-600 \mathrm{u}, 20 \mathrm{ppm}$ is achievable and, with care, $<10 \mathrm{ppm}$ is possible. However, it is not clear, for different materials, which ions should be selected or whether or not there is a significant difference between calibrations with molecular or atomic ions.

In the burgeoning fields of proteomics, accurate calibration of the mass scale is of paramount importance for the correct sequencing of peptides from protein digests. Bristow and Webb [7] have recently conducted an intercomparison of accurate mass measurement for a wide range of such spectrometers. In that study, a molecule of nominal mass 475 was supplied to 45 participants who used their local procedure. Their results show that Fourier-transform mass spectrometry (FT-MS) achieves $W<1$ ppm, TOF-based systems achieve $\sim 5 \mathrm{ppm}$, and systems involving a quadrupole combined with a TOF, between $5 \mathrm{ppm}$ and $10 \mathrm{ppm}$. These techniques have significant differences compared with SIMS. First, the ionization process is typically conducted in the gas-phase either by electron ionization, fast atom bombardment, or electrospray. Second, almost all participants used reference molecules of known mass to bracket the mass of the molecule studied to provide calibration. This was performed either simultaneously or alternately with measurement of the analyte. Of course, in SSIMS the ionization process is more energetic and one cannot bracket with reference molecules. Analysts must rely on relatively low mass ions to be correctly identified and used for calibration. This has significant benefits in making the calibration simple, requiring no additional acquisition of data or use of calibrant chemicals, but it does rely on correct identification. Furthermore, extrapolation of the mass calibration outside the range of the calibrant ions is, of course, prone to increased uncertainties. Finally, the ionization and fragmentation process in SSIMS is more complex, giving a wider spread in kinetic energies. In the following, we study these effects.

\section{Experimental}

In the present work, static SIMS analyses are made using a TOF-SIMS IV, manufactured by ION-TOF $\mathrm{GmbH}$, of the single stage reflection design [8]. The instrument is equipped with a high-resolution $\mathrm{Bi}^{+}$ focused liquid metal ion gun, incident at $45^{\circ}$ to the surface normal and operated at $25 \mathrm{keV}$ energy. The ion beam is time-focused using a pulsed buncher providing an approximately Gaussian time distribution at the surface with a FWHM of 800 ps (measured from the FWHM of $\mathrm{H}^{+}$peak). Ion arrival times are recorded using a time-to-digital converter (TDC) with a time resolution selectable between 50 ps and 1000 ps. In this study, the time resolution is set to $200 \mathrm{ps}$. This TDC is capable of registering $>1500$ ions per cycle time of
$100 \mu$. To allow voltages to settle, the instrument was allowed to warm up for $15 \mathrm{~min}$ before acquiring data.

We use one of the samples from the same stock available for the interlaboratory study $[1,3]$. The sample has a thin-layer of polycarbonate (PC) on a $1 \mathrm{~cm} \times 1 \mathrm{~cm}$ piece of clean silicon wafer. The coating and wafer are sufficiently conducting that no charge compensation is required.

\section{Characterization of a Single Stage Reflection Mass Analyzer}

Analyzer Chromatic Aberation. Here, we study in detail the single stage reflection configuration used in the ION-TOF IV [8] instrument. We first study the chromatic aberration of the analyzer, i.e., the effect of the initial spread of secondary ion kinetic energies on the measured time of flight to the detector. Figure 3 shows, schematically, the ion optical assembly modeled using the SIMION [9] ion trajectory software. In this model, a positive ion is generated at the sample surface and is rapidly accelerated by an extraction electrode at a potential of $-2000 \mathrm{~V}$ over a length $L_{\mathrm{A}}$ into the first drift region of length $L_{\mathrm{D} 1}$, and subsequently passes into an ion mirror of length $L_{R}$. After reflection by the ion mirror, the ion travels out into a second drift region of length $L_{\mathrm{D} 2}$ and is then accelerated into the micro channel plate detector. After acceleration a simple, 3-element accelerating Einzel lens is included, followed by an aperture of diameter $D$ to eliminate ions at wide angles from reaching the detector. Values for the dimensional parameters are set by deducing measurements from experimental data or through separate physical measurements. Typical flight paths are $\sim 2 \mathrm{~m}$, and typical flight times are $15 \mu$ and $65 \mu$ for ions with masses of 28 and $500 \mathrm{u}$, respectively. Electrostatic potentials are set to the known values used in the instrument. In the SIMION model, the length of the reflector $L_{\mathrm{R}}$ is adjusted

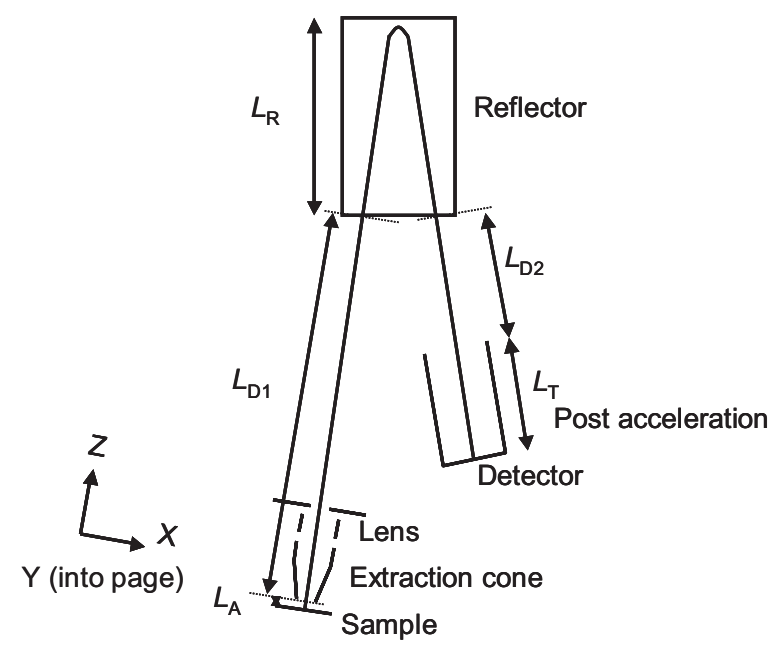

Figure 3. Schematic of the single stage reflection used in this study. The coordinate system is indicated for reference later in the text. 


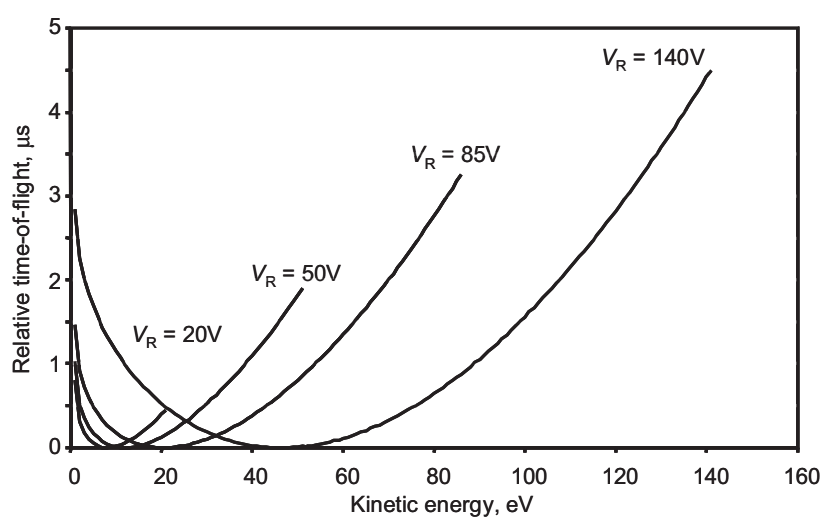

Figure 4. Relative time-of-flight for ions with energies up to $140 \mathrm{eV}$ for four reflector voltages calculated using the SIMION model. The time-of-flight is relative to the minimum flight time for each curve.

to give first-order energy compensation and the lens voltage $V_{\mathrm{L}}$ adjusted to give optimum focusing. In Figure 4 we see the effect of initial ion kinetic energy on the measured arrival time for reflector voltages $V_{\mathrm{R}}$ of $20,50,85$, and $140 \mathrm{~V}$ calculated for an ion with the mass of $\mathrm{Si}^{+}$. The spread in arrival times is parabolic with a minimum expected from first-order energy focusing conditions. The position of the minimum moves systematically to higher kinetic energy as $V_{\mathrm{R}}$ increases. Ions with a kinetic energy $>\mathrm{e} V_{\mathrm{R}}$ impact the reflector and are not detected. The curves therefore cease at a kinetic energy of $V_{R}$. Time-of-flight differences can be as high as $5 \mathrm{~ns}$ for $V_{\mathrm{R}}>140 \mathrm{~V}$, equivalent to $350 \mathrm{ppm}$ of the total time-of-flight. To investigate this behavior, we use SIMION [9] to calculate the time-of-flight for a range of initial energies as follows.

To calculate the effect of chromatic aberration on the peak shape, the ion kinetic energy distribution is required. For atomic ions, the well established collision cascade theory $[10,11]$ gives the intensity $I(E)$ for a kinetic energy, $E$, with a surface binding energy, $U$, of

$$
I(E)=\frac{E}{(E+U)^{3}}
$$

This has a maximum at $E=0.5 U$ and a high-energy tail $(E>U)$ with a characteristic $E^{-2}$ energy dependence. This function has been shown to give good fits to many sets of experimental data if $U$ is taken as a fitting parameter and not the true binding energy per atom.

The theoretical understanding of the kinetic energy distribution for molecules is not so well developed and there is significantly less experimental data available to verify theories. Theoretical approaches differ from the statistical consideration of cascades acting on sub-units of a molecule $[12,13]$ or alternatively considering the molecule as a single entity [14], which may disassociate if the internal energy is too high. The theories differ in detail but are in general agreement that, for molecules, the high-energy kinetic energy distribution has approx- imately an $E^{-5}$ energy dependence. For simplicity, here we use a modified eq 3 for molecules to give

$$
I(E)_{m o l}=\frac{E}{(E+U)^{6}}
$$

where the energy at the peak maximum, $E_{\max }$ is $U / 5$. We are not too concerned about the precise peak shape here. It is the value of $E_{\max }$ that most strongly affects the mass calibration accuracy. To illustrate this point, we calculate peak shapes for an atomic and a molecular ion and use experimental data for $E_{\max }$ to deduce realistic values for $U$. First, for the atomic ion we find an $E_{\max }$ value of $4.4 \mathrm{eV}$ for the $\mathrm{Si}^{+}$ion energy distribution with a Xe primary ion beam with energies between 3 and 30 $\mathrm{keV}$ impacting a clean silicon surface [15]. From eq 3, this gives a value of $U$ of $8.8 \mathrm{eV}$. Delcorte et al. have studied, in detail, the kinetic energy distributions of organic molecules to develop the understanding of molecular fragment emission and correlation with molecular dynamics simulation [16-18]. In an analysis of a Langmuir-Blodgett film of triacontane acid on silver [19] they show that the FWHM of the kinetic energy distributions increases with fragmentation of the molecule and the position of the peak increases in energy. For example, in the fragmentation of the molecular ion $\mathrm{C}_{3} \mathrm{H}_{7}^{+}$, successive losses of hydrogen result in $E_{\max }$ increasing from 1.41 to $3.62 \mathrm{eV}$. For illustration here, we shall use the $\mathrm{C}_{3} \mathrm{H}_{7}^{+}$ion and from eq 4 set the value of $U$ to $7.05 \mathrm{eV}$.

To study the effect of the ion kinetic energy distribution on the measured peak shape in the mass spectrum, we map the intensity distribution as a function of energy on to the time-of-flight data shown in Figure 4. We now obtain the peak shape by simply binning the intensities into time bins of 200 ps for comparison with experimental data. In addition, the peak shapes are convolved with a Gaussian function of FWHM 800 ps to take into account the ion beam pulse width in the experimental data. The final calculated peak shapes for the atomic ion $\left(\mathrm{Si}^{+}\right)$and the organic molecule $\left(\mathrm{C}_{3} \mathrm{H}_{7}^{+}\right)$are shown in Figure 5 for two reflector voltages, (Figure $5 \mathrm{a}$ ) $V_{\mathrm{R}}=5 \mathrm{~V}$ and (Figure 5b) $V_{\mathrm{R}}=80 \mathrm{~V}$.

In summary, the single stage reflector has a firstorder minimum in the flight time at a higher energy than the $E_{\max }$ of the secondary ion kinetic energy distribution for all molecules and most atomic species. Therefore, we expect the peak position in the mass spectrum to move to lower mass as $E_{\max }$ increases and not to change direction. This means that mass calibration accuracy will be strongly dependent on the choice of secondary ions used in the calibration if they have different $E_{\max }$ values. It is clear from Figure 5 that a lower reflector voltage gives the best peak shapes and smallest change in peak position. It is also clear that one may use the instrument with a high reflector voltage to give a good energy discrimination that is useful to 

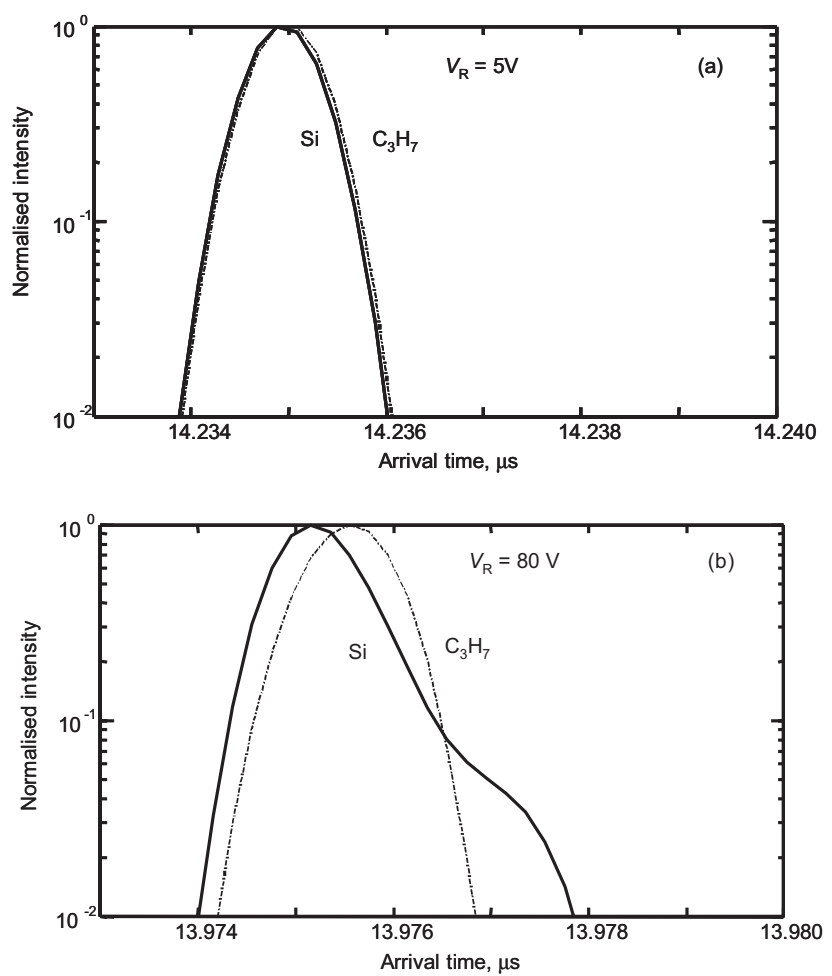

Figure 5. Calculated mass peak shapes using the kinetic energy distributions for an atomic ion ( continuous line ) and a molecular ion (dash/dot/dash line), for (a) $V_{\mathrm{R}}=5 \mathrm{~V}$, and (b) $V_{\mathrm{R}}=80 \mathrm{~V}$. The atomic ion is approximately equivalent to $\mathrm{Si}^{+}$and the molecular ion approximately equivalent to $\mathrm{C}_{3} \mathrm{H}_{7}^{+}$.

study the effect of ion kinetic energy, as we shall see later.

\section{Angular Aberration}

We now study the effect that the secondary ion angular distribution has on the measured peak shape. The angular distribution for atomic [20] and molecular [14] ions may be described by a simple $\cos (\theta)$ distribution where $\theta$ is the emission angle from the surface normal. For clarity, the X, Y, or Z coordinate orientation used is illustrated in Figure 3. For an ion emitted at an angle $\theta$ to the $Z$ axis, with velocity $\nu$, there is a component, initially $\nu \sin (\theta)$, in the plane of the sample $(X-Y)$, and this will affect the trajectory of the ion through the mass analyzer, and consequently, the time-of-flight.

Those ions emitted in the $X-Z$ plane are most strongly affected because of the larger variation of the trajectory length arising from the asymmetry of the reflector and detector about the Z-axis and consequently have the greatest range in time-of-flight. In the Y-Z plane, symmetry indicates that the first-order effect will be zero. Using the SIMION ion-trajectory model indicated earlier, we calculate the time-of-flight for a $1 \mathrm{eV}$ ion emitted for different angles in the $\mathrm{X}-\mathrm{Z}\left(\phi=0^{\circ}\right)$ and $\mathrm{Y}-\mathrm{Z}\left(\phi=90^{\circ}\right)$ planes. These data are shown in
Figure 6. Clearly, the effect is much stronger for emission in the $\mathrm{X}-\mathrm{Z}$ plane with a range of arrival times of 3.5 ns equivalent to 263 ppm- a large effect. The time-of-flight variation in the $\mathrm{Y}-\mathrm{Z}$ plane is a factor of 2 smaller. To simplify matters, we now consider only the dominating $X-Z$ plane. The effect of changing the angle of emission in the $X-Z$ plane, on the time-of-flight, increases dramatically as the ion kinetic energy is increased. As the energy increases, the time-of-flight changes dramatically because there is a higher velocity component in the dispersive direction.

A simple analysis using SIMION shows that if an aperture is placed immediately after the lens to eliminate trajectories initially $>50^{\circ}$ for $1 \mathrm{eV}$ ions, the same aperture would eliminate $12.5^{\circ}$ initial angles for $16 \mathrm{eV}$ ions. The relationship between aperture diameter $D$, emission angle $\theta$, and kinetic energy $E$, is given from the Helmholtz-Lagrange relation approximately as

$$
D \theta \sqrt{E}=\text { constant }
$$

This describes our observations and has been used to set the angular limit for each aperture size studied. Typically, the aperture is placed after the lens to eliminate the peripheral ions.

We now calculate the expected peak shape for ions of given energies using the method described to map the $\cos (\theta)$ intensity distribution with emission angle to an intensity distribution with time-of-flight. This is then converted to a mass spectrum exactly as before. The peak shapes for an ion energy of $1 \mathrm{eV}$, typical of molecular ions, and of $16 \mathrm{eV}$, typical of atomic ions, are shown in Figure 7 for a range of aperture sizes restricting angular acceptance from a fully open $90^{\circ}$ to $80^{\circ}$ and $60^{\circ}$, for an ion of $1 \mathrm{eV}$ and similarly to $1 / 4$ of these angular values for an ion of $16 \mathrm{eV}$ energy.

Clearly, the intensity decreases as the aperture size decreases but the peak shape greatly improves. Cutting out initial angles of $>60^{\circ}$ removes the shoulder on the high mass side, which gives a peak shape that relates well to experiment.

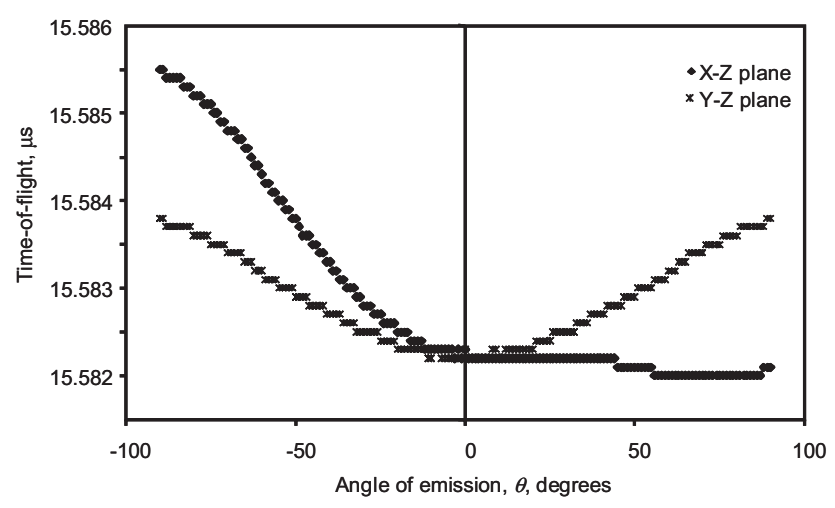

Figure 6. Time-of-flight for ions of $1 \mathrm{eV}$ kinetic energy for varying angles of emission $\theta$, (filled diamond) for emission in the $\mathrm{X}-\mathrm{Z}$ plane and (asterisk) for emission in the $\mathrm{Y}-\mathrm{Z}$ plane. 

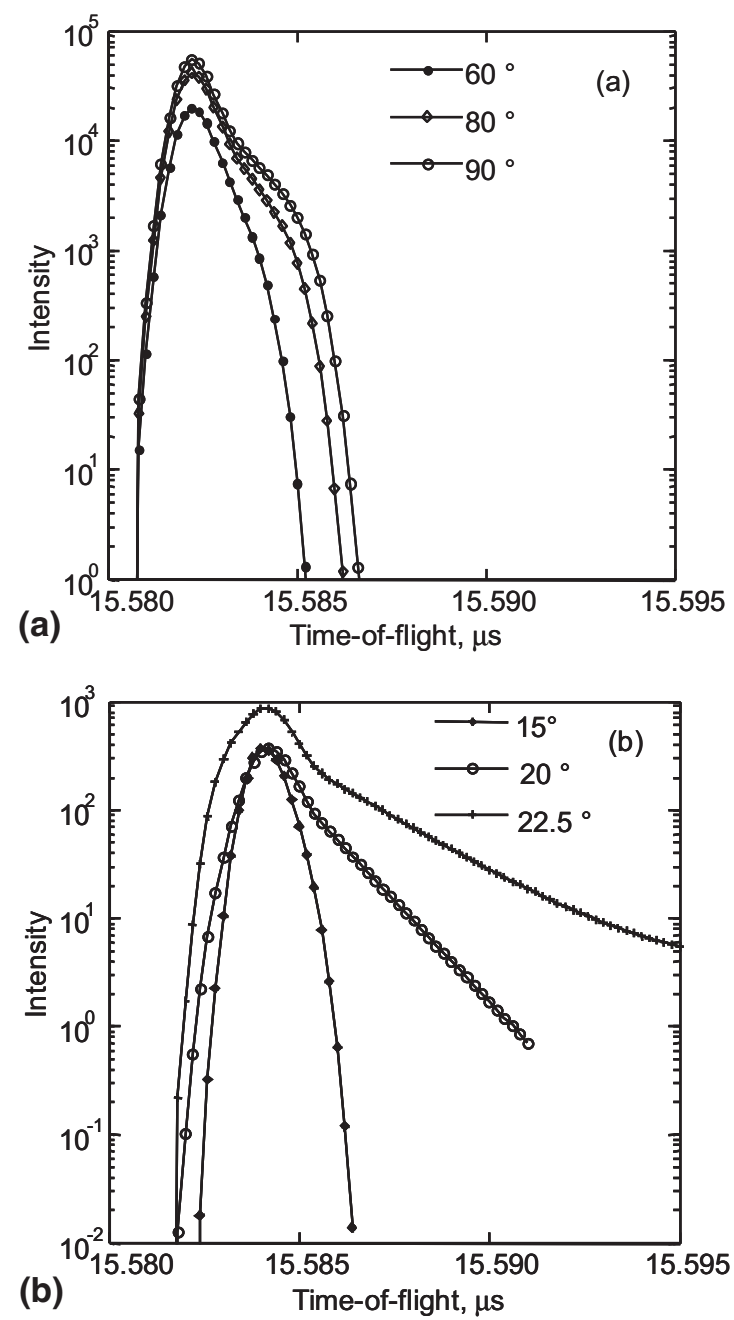

Figure 7. Peak shapes calculated using SIMION for (a) an ion of $1 \mathrm{eV}$ and $V_{\mathrm{R}}=20 \mathrm{~V}$ with angular acceptance restricted by an aperture to emission angles $\theta$ of $60^{\circ}$ (filled circle), $80^{\circ}$ (open diamond), and $90^{\circ}$ (open circle), and (b) an ion of $16 \mathrm{eV}$ and $V_{\mathrm{R}}=20 \mathrm{~V}$ with angular acceptance restricted by an aperture to the equivalent emission angles $\theta$ of $15^{\circ}(+), 20^{\circ}$ (open circle), and $22.5^{\circ}$ (filled diamond).

It should be noted that we have used no proprietary knowledge of the ion optics of the spectrometer and hence some of the details of the calculations may or may not be correct. However, the general behavior matches our observations sufficiently close to be effective. In summary, clearly the angular aberration affects the peak shape significantly and the use of an aperture greatly reduces this. Fortunately, in the mass spectrum, the peak maximum does not move significantly and so the calibration of the mass scale is relatively unaffected.

\section{Optimizing Parameters}

Before characterizing the instrumental performance we need a reliable method for the measurement of the mass values of the peaks. We have found that an asymmetric Gaussian function, $G_{\mathrm{A}}$, gives a good fit to a wide range of peak shapes where $M_{\mathrm{o}}$ is the peak centre, $M$ is the mass, and $G_{\mathrm{o}}$ is a scaling term

$$
G_{A}=G_{o} \exp \left(\frac{-\left(M-M_{o}\right)^{2}}{2\left[\sigma-\alpha\left(M-M_{o}\right)\right]^{2}}\right)
$$

and

$$
\sigma=\frac{\operatorname{FWHM}(\alpha=0)}{2 \sqrt{2 \ln 2}}
$$

Where $\operatorname{FWHM}(\alpha=0)$ is the full width at half maximum of the base Gaussian width $\alpha=0$. The term $\alpha$ gives the asymmetry and for $\alpha=0$ the function is pure Gaussian. For each peak, we fit eq 6 only to those intensities above $50 \%$ of the maximum intensity to avoid interference from neighboring peaks. Typically, the asymmetric Gaussian is an excellent description of the peak down to $15 \%$ of the maximum although the fitting only covers to $50 \%$.

To define the calibration parameters for the mass scale [21], consider a time-of-flight mass spectrometer with an effective flight path of length $L$ and of mass of an ion, $M$, with energy $E$, along the flight path. The measured arrival time referenced to the beam chopper $t$ is simply given by

$$
M=\frac{2 E\left(t-t_{0}\right)^{2}}{L^{2}}
$$

where $t_{0}$ is a delay offset to allow for the time taken for the primary ion to travel from the beam chopper to the sample. For a TOF system, two calibration coefficients $A$ and $B$ are now defined, where

$$
A=\frac{2 E}{L^{2}}
$$

and

$$
B=t_{0}
$$

such that

$$
M=A(t-B)^{2}
$$

We have ignored the extraction and reflection slopes in this simple explanation although they are included in our SIMION calculations. These change the calibrated values of $A$ and $B$ but as we calibrate $A$ and $B$ for our present purposes this is sufficient. The coefficients $A$ and $B$ are determined using least-squares fitting of the measured mass position $M_{\mathrm{o}}$ (from the fit of eq 6 to the mass peak) and the true mass $M_{\mathrm{T}}$ with the loss or gain of an electron depending on the ionization state. We shall discuss the choice of calibration ions later.

The mass accuracy, $\Delta M$ from eq 1 , for a range of molecular ions emitted from PC up to $140 \mathrm{u}$ for our 


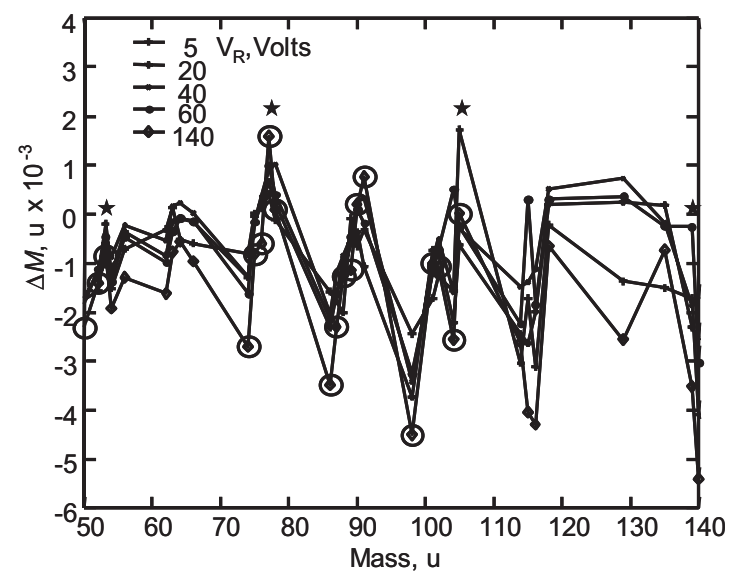

Figure 8. Mass accuracy, $\Delta M$, for hydrocarbon peaks from PC positive ion spectra with $V_{\mathrm{R}}$ values of $5 \mathrm{~V}(+), 20 \mathrm{~V}$ (asterisk), 40 $\mathrm{V}(\mathrm{x}), 60 \mathrm{~V}$ (filled circle), and $140 \mathrm{~V}$ (open diamond). The peaks denoted with a (star) are used to calibrate the spectra. The circumscribed symbols denote the mass peaks used later to measure $\sigma_{\mathrm{M}}$.

ION-TOF IV instrument is shown in Figure 8 for reflector voltages between 5 and $140 \mathrm{~V}$. The spectra are calibrated using four ions $\mathrm{C}_{4} \mathrm{H}_{5}^{+}, \mathrm{C}_{6} \mathrm{H}_{5}^{+}, \mathrm{C}_{8} \mathrm{H}_{9}^{+}$, and $\mathrm{C}_{9} \mathrm{H}_{11} \mathrm{O}^{+}$. We first note that, in general, $\Delta M$ is poorest for a high reflector voltage and that $\Delta M$ may vary by $4.3 \times 10^{-3} \mathrm{u}$ over only a few mass units. A striking feature of the data is the regular periodic behavior of $\Delta M$, starting negative and rising strongly with increasing mass over a series of mass peaks to reach a maximum value and then falling sharply again. This characteristic behavior is similar to the fragment cascades observed in G-SIMS [22] for the ratio F, of each mass intensity in a spectrum acquired with low fragmentation ion beam conditions (low effective surface plasma temperature) to those in one acquired with high fragmentation (high effective surface plasma temperature) ion beam conditions. This ratio removes ion yield or similar parameters and is dominated by changes in intensity with effective surface plasma temperature. That theory relates the population of fragment ions to a surface plasma temperature $T_{p}$, so that at lower $T_{p}$ there is a higher population of less degraded fragments. Static SIMS spectra contain a high population of fragment and degraded ions. In contrast, G-SIMS provides significantly simpler spectra, where the peaks relate directly to the parent ion structure, allowing direct interpretation and identification. This has been demonstrated for a wide range of materials [22-24]. For comparison, in Figure 9 we plot the $\Delta M$ values of Figure 8 using the most sensitive setting $\left(V_{\mathrm{R}}=140 \mathrm{~V}\right)$ alongside the fragment cascades $F$, for G-SIMS [22] for the same mass peaks. There is a good correlation between the two behaviors. From our earlier analysis of analyzer chromatic aberration, clearly those ions with a high positive $\Delta M$ value have a longer time-of-flight and therefore have a lower kinetic energy (far left of Figure 4) than those with a negative $\Delta M$ (shorter flight time). This is a characteristic of the first-order energy focus. Fragments with low kinetic energies correlate with the undegraded fragments (low $T_{\mathrm{p}}$ ) ions in G-SIMS and fragments with high kinetic energies correlate with the degraded fragments (high $T_{\mathrm{p}}$ ). This is predicted in the theory already proposed for G-SIMS [22].

Clearly, the wide range in $\Delta M$ for ions with different fragmentation has a major effect on the accuracy of the mass scale calibration. Ideally, we would like $\Delta M$ to be a flat line through zero! To provide a measure of the divergence from this we select four of the well defined $\mathrm{C}_{x} \mathrm{H}_{\mathrm{y}}^{+}$cascades with $4,6,7$, and 8 carbon atoms, respectively, as detailed in Table 1 . These ions are identified in Figure 8 by circumscribing the datapoint for the $V_{\mathrm{R}}=140 \mathrm{~V}$ dataset. We now define $\sigma_{\mathrm{M}}$ as the average of the standard deviations of $\Delta M$ for each of the four $\mathrm{C}_{x} \mathrm{H}_{\mathrm{y}}^{+}$cascades defined above. We shall use $\sigma_{\mathrm{M}}$ as a parameter to study the effect of instrument operating parameters.

First, we study the effect of reflector voltage $V_{R}$, and flight energy, $E$, as shown in Figure 10. As the flight energy reduces from 3000 to $1000 \mathrm{eV}, \sigma_{\mathrm{M}}$ increases by a factor of 2 for $V_{\mathrm{R}}=140 \mathrm{~V}$. This increase is expected because the kinetic energy spread is relatively higher compared with the flight energy. As $V_{\mathrm{R}}$ is reduced, the mass accuracy improves. In the analysis earlier we saw that the first-order energy focus for higher values of $V_{R}$ is well beyond $E_{\max }$ so that only weak energy compensation is achieved and the spread in arrival times is significantly increased. Therefore, the optimum conditions are to use the highest flight energy and lowest $V_{\mathrm{R}}$ reflector voltage. For practical reasons, a flight energy of $2 \mathrm{keV}$ and a reflector voltage of $20 \mathrm{~V}$ is a recommended compromise. High extraction fields can increase problems for insulators (in Figure 10 for a reflector voltage of $20 \mathrm{~V}$ the flight energy is not critical) and a reflector voltage set too low may be difficult to set repeatably for insulators.

Similarly, we use this procedure to optimize the potential of the central lens element to minimize $\sigma_{\mathrm{M}}$. The value of $\sigma_{\mathrm{M}}$ is sensitive to this potential, varying by a factor of 2 for an $8 \%$ change in the voltage. However,

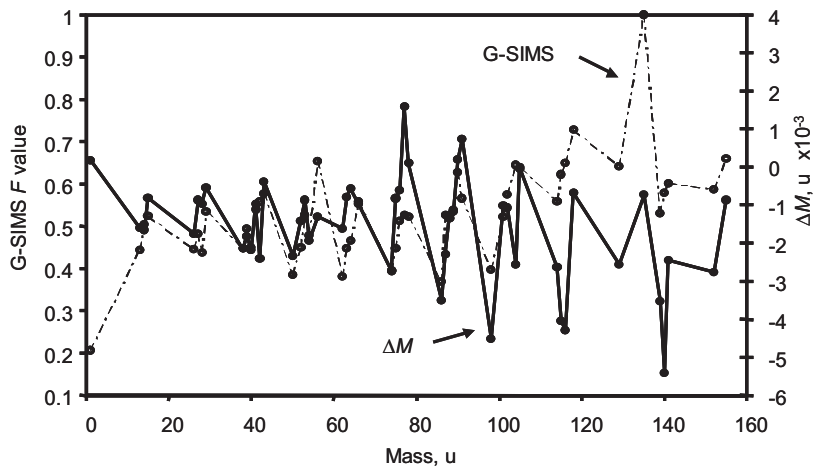

Figure 9. Comparison of the mass accuracy $\Delta M$, (filled circle) with the $F_{x}$ fragment cascade values from G-SIMS analysis using $\mathrm{Cs}$ and $\mathrm{Ar}$ ion beams (open circle) for positive ions from PC. 
Table 1. The mass accuracy for optimal instrument operating parameters, for the peaks identified in Figure 8 used to calculate $\sigma_{\mathrm{M}}$. These give a $\sigma_{\mathrm{M}}$ of $0.74 \times 10^{-3} \mathrm{u}$. In terms of the relative mass accuracy $\Delta M_{\mathrm{R}}$ this is $10 \mathrm{ppm}$

\begin{tabular}{lcccrr}
\hline Ion & True mass, $\mathrm{u}$ & Accuracy, ppm & Ion & True mass, u & Accuracy, ppm \\
\hline \hline $\mathrm{C}_{4} \mathrm{H}_{2}$ & 50.01565 & -31.2 & $\mathrm{C}_{7} \mathrm{H}_{4}$ & 88.03130 & -14.0 \\
$\mathrm{C}_{4} \mathrm{H}_{4}$ & 52.03130 & -19.1 & $\mathrm{C}_{7} \mathrm{H}_{5}$ & 89.03913 & -10.5 \\
$\mathrm{C}_{4} \mathrm{H}_{5}$ & 53.03913 & -2.7 & $\mathrm{C}_{7} \mathrm{H}_{6}$ & 90.04695 & -3.5 \\
$\mathrm{C}_{6} \mathrm{H}_{2}$ & 74.01565 & -11 & $\mathrm{C}_{7} \mathrm{H}_{7}$ & 91.05478 & -7.0 \\
$\mathrm{C}_{6} \mathrm{H}_{3}$ & 75.02348 & 1 & $\mathrm{C}_{8} \mathrm{H}_{2}$ & 98.01565 & -30.1 \\
$\mathrm{C}_{6} \mathrm{H}_{4}$ & 76.03130 & 5 & $\mathrm{C}_{8} \mathrm{H}_{5}$ & 101.03910 & -10.5 \\
$\mathrm{C}_{6} \mathrm{H}_{5}$ & 77.03913 & 8.8 & $\mathrm{C}_{8} \mathrm{H}_{6}$ & 102.04700 & -8.0 \\
$\mathrm{C}_{6} \mathrm{H}_{6}$ & 78.04695 & 4.4 & $\mathrm{C}_{8} \mathrm{H}_{8}$ & 104.06260 & -21.2 \\
$\mathrm{C}_{7} \mathrm{H}_{2}$ & 86.01565 & -26.9 & $\mathrm{C}_{8} \mathrm{H}_{9}$ & 105.07040 & -9.5 \\
$\mathrm{C}_{7} \mathrm{H}_{3}$ & 87.02348 & -20.4 & & & \\
\hline
\end{tabular}

the minimum is simple to locate. Similarly, the optimum values for the analyzer $\mathrm{X}$ and $\mathrm{Y}$ deflector plates were set. A broad minimum is observed which rises steeply for larger deflections. It is therefore important that a suitable procedure is used to align the ion optical axis and the ion beam raster area [3].

The effect of the sample distance from the extractor electrode, $L_{\mathrm{A}}$, is shown in Figure 11. At each position the lens is refocused. The optimum setting in this range is for $L_{\mathrm{A}}=1.0 \mathrm{~mm}$ where $\sigma_{\mathrm{M}}$ is lowest. A significantly lower value of $L_{\mathrm{A}}$ is impractical since the beam then strikes the extractor and, additionally, the higher extraction field at small gaps may cause problems for insulating samples or samples with high surface topography. We expect the value of $\sigma_{\mathrm{M}}$ to be proportional to the increased length of the off-axis trajectory squared and hence $\sigma_{\mathrm{M}}$ will be proportional to $L_{\mathrm{A}}^{2}$. The line through the data in Figure 11 is this function. For practical reasons we shall use an extraction gap $L_{\mathrm{A}}$ of $1.5 \mathrm{~mm}$ in the rest of this work.

In summary, for this instrument, for the smallest variation in mass positions for peaks with different kinetic energies, we recommend a flight energy $E$ of $2000 \mathrm{eV}$, a reflector voltage $V_{\mathrm{R}}$ of $20 \mathrm{~V}$, an extraction gap $L_{\mathrm{A}}$ of $1.5 \mathrm{~mm}$, and an optimization procedure to ensure

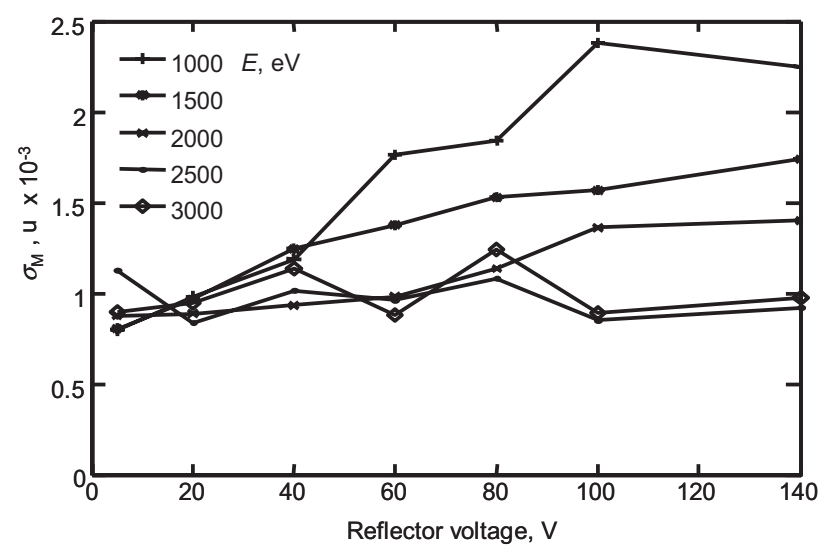

Figure 10. Mass accuracy deviation, $\sigma_{\mathrm{M}}$, for a range of reflector voltage values and for ion flight energies of $1000 \mathrm{eV}(+), 1500 \mathrm{eV}$ (asterisk), $2000 \mathrm{eV}$ (x), $2500 \mathrm{eV}$ (filled circle), and $3000 \mathrm{eV}$ (open diamond). the best focus condition of the lens and setting of the analyzer deflection plates. These conditions have been used throughout this study unless stated otherwise. Table 1 shows the mass accuracy for the peaks used to define $\sigma_{\mathrm{M}}$ with the above recommended operating parameters. This gives a value of $\sigma_{\mathrm{M}}$ of $0.74 \times 10^{-3} \mathrm{u}$. In terms of the relative accuracy, $\Delta M_{\mathrm{R}}$ is $10 \mathrm{ppm}$. The single stage reflection design with first-order energy focusing seems to limit further improvement. To improve this further, better energy compensation is required with a minimum that is flat through $0 \mathrm{eV}$. In the interlaboratory study we see that the mass accuracy of instruments with second-order energy focusing is a factor of 1.8 better.

\section{Calibration Procedure}

So far, we have defined the optimum instrument operating parameters which give an average mass accuracy for all molecules from PC in the range 0 to $140 \mathrm{u}$ of $10 \mathrm{ppm}$. We have also identified the reasons why atomic and high-energy fragments have lower measured peak masses than the true mass position and so are not recommended for use in a calibration procedure for molecular ions. Earlier, we saw from the interlaboratory study, using in-house procedures, that

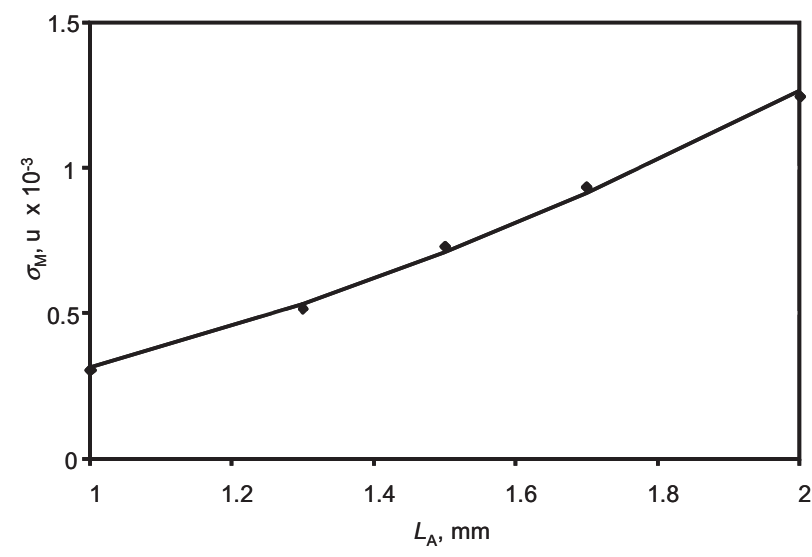

Figure 11. The effect of distance between sample surface and the extraction electrode, $L_{\mathrm{A}}$, on the mass accuracy deviation $\sigma_{\mathrm{M}}$. The line through the data shows the function $\sigma_{\mathrm{M}}$ proportional to $L_{\mathrm{A}}^{2}$. 
the mass accuracy for the Irgafos 168 protonated molecular ion at mass $647.459 \mathrm{u}$ has poor relative mass accuracy $\Delta M_{\mathrm{R}}$ of $-150 \mathrm{ppm}$.

We now study the effect of the mass values of the calibration ions and the accuracy of the calibration extrapolation to high mass ions. If there were no uncertainties in the system, there would be no extrapolation error beyond the calibration mass range. However, as we have already seen the ion kinetic energy causes a significant effect on the measured mass. From Figure 8 we see that over a fragmentation series, for example $\mathrm{C}_{6} \mathrm{H}_{6}^{+}$to $\mathrm{C}_{6} \mathrm{H}_{2}^{+}$with successive loss of one hydrogen atom, the increase in kinetic energy results in a negative mass difference of approximately $\Delta M=-3 \times 10^{-3} \mathrm{u}$, for a typical reflector voltage of $20 \mathrm{~V}$. As a first approximation, we shall use this value as a constant uncertainty in mass position, $U_{0}$, for ions with typical kinetic energy across the mass range.

In the simplest case, the combined uncertainty $U(m)$, for a calibration using two peaks at masses $m_{1}$ and $m_{2}$, is given by [25]

$$
U(m)=\left[\left(\frac{m_{2}-m}{m_{2}-m_{1}}\right)^{2} U_{1}^{2}+\left(\frac{m-m_{1}}{m_{2}-m_{1}}\right)^{2} U_{2}^{2}\right]^{1 / 2}
$$

For practical purposes $U_{1}=U_{2}=U_{0}$ over most of the mass range of importance here. When $m$ is equal to $m_{1}$ or $m_{2}$, this function gives $U(m)=U_{0}$ as expected from least-squares fitting and when $m$ is half way between $m_{1}$ and $m_{2}$ then $U(m)=U_{0} / \sqrt{ } 2$. Figure 12 shows the relative uncertainty $U / U_{0}$ using eq 12 for five separate calibrations with $m_{1}=10$ and $m_{2}$ varying between 100 and 2000. Clearly, the calibration uncertainty rises rapidly outside the calibration mass interval and, for a typical calibration interval with $m_{1}=10$ and $m_{2}=100$, we find $U / U_{0}=20$ at $m=1000 \mathrm{u}$, which is equivalent to a relative mass accuracy $W$ of $-60 \mathrm{ppm}$ (if $U_{0}=-3$ $\times 10^{-3} \mathrm{u}$ ). This is smaller than the average value from the interlaboratory study but is consistent with the instruments exhibiting better performance.

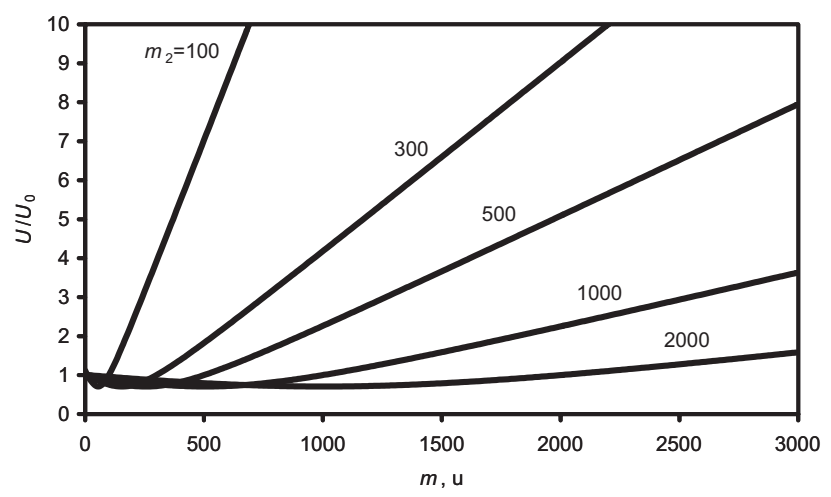

Figure 12. Relative uncertainties, $U / U_{0}$, using two calibration mass peaks illustrating the effect of extrapolation as a function of the given mass peak, $m$, up to $3500 \mathrm{u}$ with $m_{1}=10$ and with separate curves for $m_{2}$ set at 100, 300, 500, 1000, and $2000 \mathrm{u}$.
The requirement to use widely separated masses in the calibration is illustrated in Figure 12. To ensure that the total uncertainty is not too high (say, $U / U_{0}<2$ ) for accurate mass measurement of large molecules of mass $m$, the selected calibration ions should include $m_{2} \geq 0.55 \mathrm{~m}$.

It is common practice to use hydrogen as $m_{1}$ in the calibration. This is very useful as it is easy to identify without a calibrated mass scale and may be used to establish a first calibration. However, we then find $U_{1}$ significantly greater than $U_{0}$, for accurate calibration of the mass scale so $\mathrm{H}^{+}$is not recommended. Part of the reason for the higher value of $U_{1}$ is that the trajectory of hydrogen is affected more strongly by magnetic fields than heavier ions (often observed as a displacement of the ion image), thus adding to the uncertainty in the measured mass value. We conclude from this analysis that a practical and optimal calibration scheme would use a mass $m_{1}$ around 12 to $30 \mathrm{u}$ and with $m_{2}$ at as high a value as conveniently available. For molecular analysis of minimally degraded fragments, it is best to use peaks for similarly minimally degraded entities. To check the linearity of the calibration and to reduce the reliance on any individual peak, (which may have an uncertainty higher than $U_{0}$ ), further intermediate calibration masses may be added.

\section{Conclusions}

We have presented a detailed analysis of the factors affecting the calibration of the mass scale for a popular single stage reflection TOF-SIMS instrument. Detailed models using the ray tracing software SIMION are used to study the effect of ion kinetic energy-chromatic aberration and the effect of angular emission on the time-of-flight. This shows that molecules with a low kinetic energy have a significantly longer time-of-flight than those with higher kinetic energies because the first-order minimum in the flight time is not coincident with the kinetic energy maximum for most molecular fragments. For better performance, a second-order energy focusing is required at the appropriate energy. A procedure based on reducing the spread of time-offlights for selected ions with different kinetic energy is given.

In summary, for establishing a mass scale for accurate mass measurement of molecules we recommend: (1) using the procedure described to optimize the instrument parameters to reduce $\sigma_{\mathrm{M}}$; (2) calibration using ions that have low degradation or fragmentation from the original parent structure, these may be identified using G-SIMS [22]; (3) for the analysis of molecules, do not include atomic ions; (4) not including hydrogen in a final calibration; and (5) selecting a mass range of calibration ions to give the required accuracy for large molecules using Figure 12 as a guide, including a mass greater then $55 \%$ of the peak to be assigned. 


\section{Acknowledgments}

This work forms part of the Valid Analytical Measurement Program of the National Measurement System of the UK Department of Trade and Industry. The authors are grateful to Dr. T. Bristow and Dr. G. O'Connor of LGC for helpful discussions about gas-phase mass spectrometry.

\section{References}

1. Gilmore, I. S.; Seah, M. P.; Green, F. M. Static TOF-SIMS-A VAMAS Interlaboratory Study. Part I. Repeatability and Reproducibility of Spectra. Surf. Interface Anal. 2005, 37, 651-672.

2. International Standards Organization www.iso.org.

3. Gilmore,, I. S., Seah,, M. P. VAMAS 2002: Static TOF SIMS Interlaboratory Study-Protocol for Analysis. NPL Report 2001, COAM(A)6.

4. Niehuis, E.; Van Velzen, P. N. T.; Lub, J.; Heller, T.; Benninghoven, A. High Mass Resolution Time-of-Flight Secondary Ion Mass Spectrometry. Application to Peak Assignments. Surf. Interface Anal. 1989, 14, $135-142$.

5. Niehuis, E. Exact Mass Determination Using TOF-SIMS. In SIMS VII; John Wiley and Sons, Chichester, 1990; pp 299-304.

6. Reichlmaier, S.; Hammond, J. S.; Hearn, M. J.; Briggs, D. Analysis of Polymer Surfaces by SIMS 17. An Assessment of the Accuracy of the Mass Assignment Using a High Mass Resolution TOF-SIMS Instrument. Surf. Interface Anal. 1994, 21, 739-746.

7. Bristow, A. W. T.; Webb, K. S. Intercomparison Study on Accurate Mass Measurement of Small Molecules in Mass Spectrometry. J. Am. Soc. Mass Spectrom. 2003, 14, 1086-1098.

8. Schwieters, J.; Cramer, H. G.; Heller, T.; Jürgens, U.; Niehuis, E.; Zehnphenning, J.; Benninghoven, A. High Mass Resolution Surface Imaging with a Time-of-Flight Secondary Ion Mass Spectroscopy Scanning Microprobe. J. Vac. Sci. Technol. A 1991, 9, 2864-2871.

9. Dahl,, D. A. SIMION 3D, version 7.0; Idaho National Engineering and Environmental Laboratory.
10. Sigmund, P. Sputtering by Ion Bombardment: Theoretical Concepts. In Sputtering by Particle Bombardment I, Behrisch, R., Ed.; Springer: Berlin, 1981, p 37.

11. Thompson, M. W. The Energy Spectrum of Ejected Atoms during the High Energy Sputtering of Gold. Philos. Mag. 1968, 18, 377-414.

12. Urbassek, H. M. Sputtering of Molecules. NIMB 1987, 18, 587-595.

13. Hoogerbrugge, R.; Kistemaker, P. G. Kinetic and Internal Energy Distributions of Sputtered Molecules and Clusters. NIMB 1987, 18, 600-604.

14. Haring, R. A.; Roosendaal, H. E.; Zalm, P. C. On the energy and angular distribution of sputtered polyatomic molecules. NIMB 1987, 28, 205-213.

15. Wittmaack, K. Secondary-Ion Emission from Silicon Bombarded with Atomic and Molecular Noble-Gas Ions. Surf. Sci. 1979, 90, 557-563.

16. Delcorte, A.; Bertrand, P. Kinetic Energy Distributions of Secondary Molecular Ions from Thin Organic Films Under Ion Bombardment. NIMB 1996, 115, 246-250.

17. Delcorte, A.; Bertrand, P. Energy Distributions of Hydrocarbon Secondary Ions from Thin Organic Films Under keV Ion Bombardment: Correlation Between Kinetic and Formation Energy of Ions Sputtered from Tricosenoic Acid. NIMB 1996, 117, 235-242.

18. Delcorte, A.; Vanden Eynde, X.; Bertrand, P.; Vickerman, J. C.; Garrison, B. J. Kiloelectron Volt Particle-Induced Emission and Fragmentation of Polystyrene Molecules Adsorbed on Silver: Insights from Molecular Dynamics. J. Phys. Chem. B 2000, 104, 2673-2691.

19. Delcorte, A. Fundamental Aspects of Organic SIMS. In TOF-SIMS Surface Analysis by Mass Spectrometry, Vickerman, J. C.; Briggs, D., Eds.; Surface Spectrum: UK, 2001; pp 161-195.

20. Snowdon, K. J.; Haring, R. A. Diatomic Molecule Sputtering in the Independent Binary Collision Approximation. NIMB 1987, 18, 596-599.

21. ISO 22048:2004-Surface Chemical Analysis-Information format for Static SIMS.

22. Gilmore, I. S.; Seah, M. P. Static SIMS: Towards Unfragmented Mass Spectra-The G-SIMS Procedure. Appl. Surf. Sci. 2000, 161, 465-480.

23. Gilmore, I. S.; Seah, M. P. G-SIMS of Crystallizable Organics. Appl. Surf. Sci. 2003, 203/204, 551-555

24. Gilmore, I. S.; Seah, M. P. Organic Molecule Characterization-G-SIMS. Appl. Surf. Sci. 2004, 231/232, 224-229.

25. Seah, M. P.; Gilmore, I. S.; Spencer, S. J. XPS. Binding Energy Calibration of Electron Spectrometers 4-Assessment of Effects for Different X-ray Sources, Analyzer Resolutions, Angles of Emission, and of the Overall Uncertainties. Surf. Interface Anal. 1998, 26, 617-641. 